\title{
Labetalol-Induced Hepatotoxicity during Pregnancy: A Case Report
}

\author{
Anna Whelan, $\mathrm{MD}^{1}$ Joanna Izewski, $\mathrm{MD}^{1}$ Charles Berkelhammer, $\mathrm{MD}^{2}$ Jami Walloch, $\mathrm{MD}^{3}$ \\ Helen H. Kay, MD 4 \\ ${ }^{1}$ Department of Obstetrics and Gynecology, University of Illinois \\ College of Medicine at Chicago, Chicago, Illinois \\ ${ }^{2}$ Department of Gastroenterology, Advocate Christ Medicine Center, \\ Oak Lawn, Illinois \\ ${ }^{3}$ Department of Pathology, Advocate Christ Medical Center, Oak \\ Lawn, Illinois \\ ${ }^{4}$ Department of Maternal-Fetal Medicine, Advocate Christ Medical \\ Center, Oak Lawn, Illinois \\ Am J Perinatol Rep 2020;10:e210-e212. \\ Address for correspondence Anna Whelan, MD, Department of \\ Obstetrics and Gynecology, University of Illinois College of Medicine \\ at Chicago, 840 S. Wood Street, M/C 808, Chicago, IL 60612 \\ (e-mail: Anna.whelan.md@gmail.com).
}

\author{
Abstract \\ Keywords \\ - labetalol \\ - hypertension in \\ pregnancy \\ - hepatotoxicity \\ - transaminitis
}

Background Drug-induced liver injury is a common cause of transaminitis, occurring in up to $5 \%$ of patients who are hospitalized for liver failure. In pregnancy, transaminitis is seen in conditions which may require expedited delivery.

Case A 39-year-old G2P0010 at 27 2/7 weeks' gestation with chronic hypertension on labetalol was found to have elevated transaminases. Evaluation for preeclampsia, acute fatty liver, nonalcoholic steatohepatitis, cholelithiasis, infections, and autoimmune conditions were all negative. Labetalol was then discontinued, and liver biopsy was performed. After discontinuation of labetalol, her hepatitis improved, and she was discharged on hospital day 12 and went on to deliver at term.

Conclusion Labetalol-induced hepatitis should be considered in the differential for transaminitis during pregnancy to prevent iatrogenic preterm delivery.
Transaminitis in pregnancy is a serious and ominous finding for clinicians. It is often a sign of severe preeclampsia, hemolysis, elevated liver enzymes, and low platelets (HELLP) syndrome or acute fatty liver of pregnancy (AFLP). When present, these can lead to severe maternal and neonatal consequences. However, in the absence of other signs and symptoms of these diseases, it is important to examine other potential causes of hepatotoxicity.

Drug-induced liver injury is an often overlooked, yet fairly common cause of hepatic damage. As many as $20 \%$ of patients who are hospitalized for symptoms of liver failure in the United States can be attributed to medication exposure. ${ }^{1,2}$ The overall incidence of drug-induced liver injury is approximately 1 in 10,000 to 1 in 100,000 patients. $^{2,3}$

Labetalol is a selective $\alpha$-adrenergic antagonist and nonselective $\beta$-adrenergic antagonist that is commonly used in the treatment of hypertension. It has an excellent safety profile and is one of the first-line medications to treat hypertension in pregnancy. ${ }^{4}$ Common side effects reported include orthostatic hypotension, headache, dizziness, and nausea. ${ }^{1-3}$ Serious side effects may include severe bradycardia, hypotension, cardiac impairment, bronchospasm, respiratory distress, and hypoglycemia. ${ }^{5}$ But since the 1980 s, there have been few reports underlining serious hepatic injury in patients taking labetalol. ${ }^{1,6-8}$ Due to its rarity, physicians may be unaware of this possible cause of hepatotoxicity.

\section{Case Report}

A 39-year-old G2P1011 at 272/7 weeks' gestation with a history of chronic hypertension and myomectomy presented to the emergency room with severe left lower quadrant pain. She denied headache, blurred vision, shortness of breath, or right upper quadrant pain. Her vital signs were normal received

February 15, 2020

accepted

March 7, 2020
DOI https://doi.org/

10.1055/s-0040-1713789.

ISSN 2157-6998.
Copyright $\odot 2020$ by Thieme Medical

Publishers, Inc., 333 Seventh Avenue, New York, NY 10001, USA.

Tel: +1(212) 760-0888.
License terms

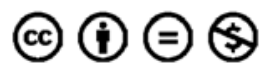


except for blood pressure of 159/92. Exam showed tenderness to palpation in the left lower quadrant, normal neurologic exam, and normal uterine and cervical exam. Fetal heart tracing was reassuring.

After arrival, blood pressures were elevated with some in the severe range, 144 to 173 systolic over 92 to 109 diastolic. Review of the patient's prenatal records indicated that she had been started on labetalol 1 month prior to conception. She required intravenous labetalol $20 \mathrm{mg}$ once and was restarted on prescribed labetalol dose of $200 \mathrm{mg}$ twice a day, although she reported taking only $100 \mathrm{mg}$ twice a day, and had not taken any medication for 3 days prior to presentation. She had elevated liver enzymes with alanine transaminase (ALT) of $206 \mathrm{U} / \mathrm{L}$ and aspartate transaminase (AST) of $524 \mathrm{U} / \mathrm{L}$. Alkaline phosphatase was normal at $86 \mathrm{U} / \mathrm{L}$ and total bilirubin was normal at $0.5 \mathrm{mg} / \mathrm{dL}$. The urine protein/creatinine ratio was 171 . An ammonia level was sent to assess for fatty liver of pregnancy and was normal at $14 \mu \mathrm{mol} / \mathrm{L}$. Blood smear showed no signs of hemolysis and lactate dehydrogenase was normal. The patient denied alcohol or acetaminophen use or abuse. She was admitted for further evaluation of her elevated transaminases. Delivery was held due to her overall stable clinical picture which did not support a diagnosis of severe preeclampsia.

Extensive serological testing for causes of hepatitis were negative including: hepatitis $\mathrm{A} / \mathrm{B} / \mathrm{C} / \mathrm{E}$, cytomegalovirus, Epstein-Barr virus, herpes simplex virus, autoimmune hepatitis (antinuclear antibodies, smooth muscle antibodies, antineutrophil cytoplasmic antibodies, lupus anticoagulant, liver-kidney microsomal antibodies, antiphospholipid antibodies), and bile acids. Liver ultrasound scan and magnetic resonance imaging showed no evidence of hepatic necrosis or fatty liver. Doppler ultrasound showed patent hepatic veins and echocardiogram was normal.
On hospital day 4, with transaminase levels still rising and all testing negative to date, labetalol was discontinued, even though she was on a relatively low dose at $200 \mathrm{mg}$ Q12 hours, and she was switched to nifedipine for her hypertension. Her left lower quadrant pain was attributed to a degenerating $5 \mathrm{~cm}$ myoma and improved during hospitalization. On hospital day 8, her transaminases peaked with an AST of $360 \mathrm{U} / \mathrm{L}$ and ALT of 1,099 U/L. Due to the uncertainty of her diagnosis, the patient underwent a liver core biopsy without complications. Over the next 4 days, her AST and ALT downtrended (-Fig. 1). At discharge on hospital day 12, her AST improved to $215 \mathrm{U} / \mathrm{L}$ and ALT to $754 \mathrm{U} / \mathrm{L}$.

The liver biopsy, with input from consulting hepatopathologists, was reported as normal liver architecture without fibrosis, steatosis, or cholestasis (-Fig. 2). No viral inclusions were observed, and inflammatory cells were predominantly mononuclear without large numbers of eosinophils. There was no evidence for autoimmune hepatitis, HELLP or AFLP. No centrilobular necrosis was observed which would be observed with acetaminophen toxicity. The diagnosis of drug-induced liver injury was made. Prompt improvement in liver enzymes after discontinuation of labetalol supported this diagnosis. The patient subsequently presented at term 9 weeks later and underwent a normal delivery. Liver enzymes by that time had improved to near normal with AST of $42 \mathrm{U} / \mathrm{L}$ and ALT of $83 \mathrm{U} / \mathrm{L}$.

\section{Discussion}

Due to the overall safety profile of labetalol and the rarity of idiosyncratic reactions, it is currently one of the first-line drugs used to treat hypertension in pregnancy. ${ }^{4}$ We describe a case of labetalol-induced hepatitis occurring in pregnancy. We believe that this case report is important as a reminder that there may be other explanations for transaminitis in a pregnant patient

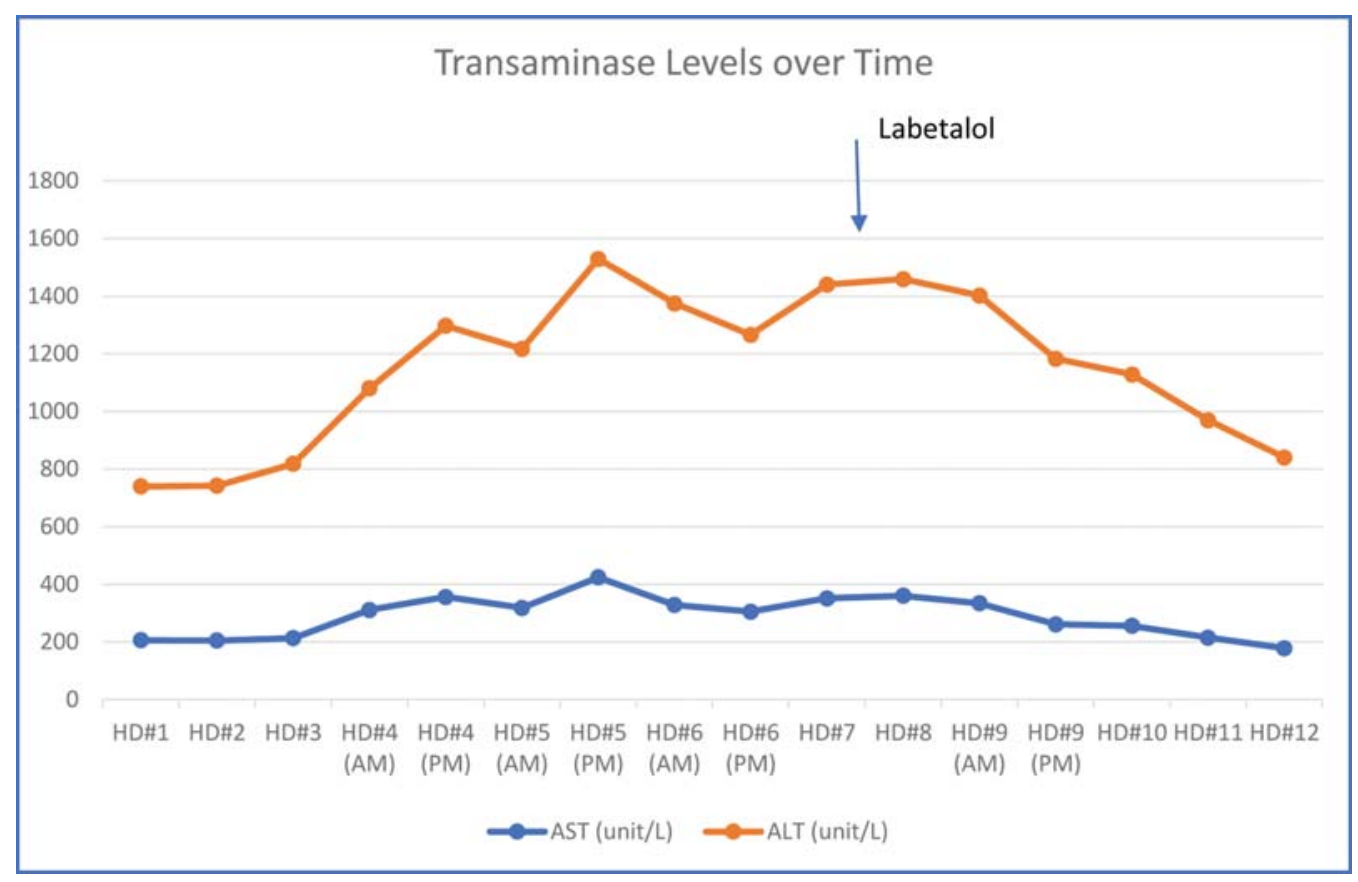

Fig. 1 Trend of transaminase levels from admission to hospital discharge. 


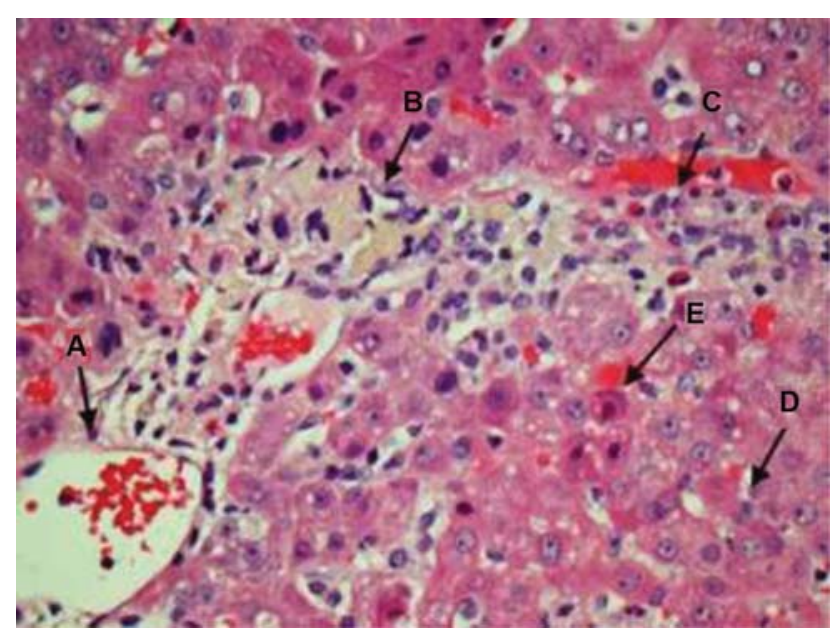

Fig. 2 Liver biopsy: The histologic features include a central vein (A), debris-laden macrophages (B), admixed with eosinophils (C), the sinusoids with increased Kupffer cells with debris (D), and rare acidophil bodies-eosinophilic necrotic hepatocytes (E).

aside from preeclampsia, HELLP or AFLP. Our patient was spared a potential iatrogenic preterm delivery when a thorough investigation suggested labetalol toxicity.

Labetalol is primarily cleared in the liver. It is rapidly taken up and approximately 80 to $90 \%$ of the drug in blood is removed in a single pass. ${ }^{6}$ Labetalol can cause transaminitis and in rare occasions may lead to significant hepatotoxicity, hepatocellular injury, hepatocellular necrosis, liver failure, and death. $^{1-3,7,8}$ The hepatotoxicity is most often considered an idiosyncratic response due to a presentation at therapeutic doses along with an indolent and variable latency period which can range from 5 to 90 days. ${ }^{2}$ Idiosyncratic drug reactions account for approximately $20 \%$ of cases with liver injury severe enough for hospitalization and occur more commonly in women than men. ${ }^{2}$ In a multicenter open label trial, labetalol was shown to cause reversible asymptomatic transaminase elevations in $8 \%$ of patients using the drug. ${ }^{7}$ Some of these patients were rechallenged with the drug and again showed an elevation in transminases. ${ }^{7}$ Since case reports emerged in the 1980s, the hepatotoxicity of labetalol has been documented in multiple reports. ${ }^{2,3,7,8}$ This is significant, as rarely this can progress to hepatic failure in pregnancy. ${ }^{8}$

The pathogenesis of labetalol hepatotoxicity is not completely known but is attributed to several intracellular pathways, of which one is the mitochondrial cytochrome P450 enzymatic pathway. Patients with isoenzyme differences or mutations in this enzyme gene may be more susceptible to its hepatotoxic effects. Other mechanisms may include an autoimmune and inflammatory response., 3,8

Liver biopsies in patients diagnosed with labetalol hepatoxicity typically show scant amounts of lymphocytes and diffuse findings of variable degrees of necrosis and apoptosis. In contrast, specimens from patients with autoimmune or infectious hepatitis show lymphocytic infiltration. ${ }^{3}$ It has been argued that the hepatotoxicity from labetalol may represent an allergic reaction. However, the lack of eosinophils in our patient's biopsy, as well as the indolent time course makes an allergic etiology less likely. ${ }^{3}$ Our patient's biopsy was consistent with idiosyncratic drug toxicity in that it showed "sparse" inflammatory cells without other overwhelming etiologies such as centrilobular necrosis due to acetaminophen toxicity or intracellular fat deposits due to nonalcoholic steatohepatitis.

Labetalol liver damage can occur even at average doses of $200 \mathrm{mg} / \mathrm{d}$ (range from 50-600 mg daily). Our patient had been prescribed a dose of $200 \mathrm{mg}$ twice daily, but was taking less than that per her admission. Patients with hepatotoxicity most commonly present with a combination of jaundice and anorexia. ${ }^{2,3}$ In reported cases, ALT and AST can rise 10 to 100 times from baseline. ${ }^{2,3,7,8}$

Although there is no practical means to prevent labetalol toxicity, aside from awareness and close monitoring, we recommend that all patients with chronic hypertension undergo baseline liver evaluation with complete blood count and a comprehensive metabolic panel either preconception or at their first prenatal visit. It is important to assess their baseline transaminase levels prior to starting labetalol during pregnancy for patients with hypertension.

\section{Conclusion}

Drug-induced liver injury secondary to the use of labetalol is rare but a well-described reaction in the general, nonpregnant population. Its presentation may be confused with other diseases in pregnancy which can lead to iatrogenic preterm delivery. It is important to include drug-induced liver injury in the differential when assessing pregnant patients with elevated liver enzymes.

\section{Disclosure}

The authors have nothing to disclose.

\section{Note}

This case report was presented as a poster at the ACOG 2019 Annual Meeting on May 3-6, 2019, in Nashville, TN.

Conflict of Interest

None declared.

\section{References}

1 Stronkhorst A, Bosma A, van Leeuwen DJ. A case of labetalolinduced hepatitis. Neth J Med 1992;40(3-4):200-202

2 Lee WM. Drug-induced hepatotoxicity. N Engl J Med 2003;349 (05):474-485

3 Douglas DD, Yang RD, Jensen P, Thiele DL. Fatal labetalol-induced hepatic injury. Am J Med 1989;87(02):235-236

4 ACOG Practice Bulletin No. 203: chronic hypertension in pregnancy. Obstet Gynecol 2019;133(01):e26-e50

5 Grassin Delyle S, Duverneuil-Mayer C, Abe E, et al. Fatal intoxication with labetalol (Trandate). Forensic Sci Int 2008;178(2-3): e19-e21

6 McNeil JJ, Louis WJ. Clinical pharmacokinetics of labetalol. Clin Pharmacokinet 1984;9(02):157-167

7 Clark JA, Zimmerman HJ, Tanner LA. Labetalol hepatotoxicity. Ann Intern Med 1990;113(03):210-213

8 Long RC, Wofford MR, Harkins KG, Minor DS. Hepatocellular necrosis associated with labetalol. J Clin Hypertens (Greenwich) 2007;9(04):287-290 